\title{
Development of an Explainable Clinical Decision Support System for the Prediction of Patient Quality of Life in Amyotrophic Lateral Sclerosis
}

\author{
Anna Markella Antoniadi \\ FutureNeuro SFI Research Centre, \\ School of Computer Science, \\ University College Dublin \\ Dublin, Ireland \\ anna.antoniadi@ucdconnect.ie
}

\author{
Miriam Galvin \\ Academic Unit of Neurology, \\ Trinity Biomedical Sciences Institute, \\ Trinity College Dublin \\ Dublin, Ireland \\ galvinmi@tcd.ie
}

\author{
Mark Heverin \\ Academic Unit of Neurology, \\ Trinity Biomedical Sciences Institute, \\ Trinity College Dublin \\ Dublin, Ireland \\ mark.heverin@tcd.ie
}

\author{
Orla Hardiman \\ Academic Unit of Neurology, \\ Trinity Biomedical Sciences Institute, \\ Trinity College Dublin \\ Dublin, Ireland \\ hardimao@tcd.ie
}

\author{
Catherine Mooney \\ FutureNeuro SFI Research Centre, \\ School of Computer Science, \\ University College Dublin \\ Dublin, Ireland \\ catherine.mooney@ucd.ie
}

\begin{abstract}
Amyotrophic Lateral Sclerosis (ALS) is a rare neurodegenerative and currently incurable disease. It causes a rapid decline in motor functions and has a fatal trajectory. The aim of the treatment is mostly to alleviate symptoms and improve the patient's quality of life (QoL). The goal of this study is to develop a Clinical Decision Support System (CDSS) in order to alert clinicians when a patient is at risk of experiencing a low QoL, so that they are better supported. The source of the data was the Irish ALS Registry and interviews with the 90 patients and their primary informal caregiver at three time-points. In this dataset, there were two different scores to measure a person's overall QoL, based on the McGill QoL (MQoL) Questionnaire and we worked towards the prediction of both. The method we used for the development of the predictive models was Extreme Gradient Boosting (XGBoost), which was compared to a logistic regression baseline model. We used the SHAP (SHapley Additive exPlanations) values as a technique to provide local and global explanations to the outputs as well as to select the most important features. The total calculated MQoL score was predicted accurately by three features, with a F1-score on the test set equal to 0.81 , a recall score of 0.78 , and a precision score of 0.84 , while, the addition of two features produced similar outcomes $(0.79,0.70$ and 0.90 respectively). The three most important features were the age at disease onset, ALSFRS score for orthopnoea and the caregiver's status pre-caregiving.
\end{abstract}

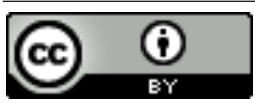

This work is licensed under a Creative Commons Attribution International 4.0 License. SAC '21, March 22-26, 2021, Virtual Event, Republic of Korea

(C) 2021 Copyright held by the owner/author(s).

ACM ISBN 978-1-4503-8104-8/21/03.

https://doi.org/10.1145/3412841.3441940

\section{CCS CONCEPTS}

- Computing methodologies $\rightarrow$ Supervised learning by classification; $\bullet$ Applied computing $\rightarrow$ Health informatics;

\section{KEYWORDS}

Amyotrophic Lateral Sclerosis, Quality of Life, Clinical Decision Support System, Machine Learning, Explainable Artificial Intelligence

\section{ACM Reference Format:}

Anna Markella Antoniadi, Miriam Galvin, Mark Heverin, Orla Hardiman, and Catherine Mooney. 2021. Development of an Explainable Clinical Decision Support System for the Prediction of Patient Quality of Life in Amyotrophic Lateral Sclerosis. In The 36th ACM/SIGAPP Symposium on Applied Computing (SAC '21), March 22-26, 2021, Virtual Event, Republic of Korea. ACM, New York, NY, USA, Article 4, 9 pages. https://doi.org/10.1145/3412841. 3441940

\section{INTRODUCTION}

Amyotrophic Lateral Sclerosis (ALS), also known as Motor Neuron Disease (MND) is a rare neurodegenerative disease. It is characterised by the progressive death of upper and lower motor neurons, causing muscle atrophy and paralysis, and it has a fatal trajectory usually within 3-4 years from symptom onset[31]. As ALS is currently incurable, treatment is mostly palliative and aims to alleviate symptoms and improve quality of life (QoL) [21, 34].

Knowledge of the determinants of a patient's QoL is of interest to clinicians as they can better guide their treatment and support. However, QoL may depend on several characteristics in different combinations according to each individual, including but not limited to the patient's physical disability. These combinations and interactions between the factors relating to QoL may be complicated for a clinician to identify. Machine Learning allows for the identification of such interactions and the prediction of an outcome of interest, which can be "learned" from the analysis of large volumes of data. For this reason it is used in different aspects of healthcare, such as 
in the development of Clinical Decision Support Systems (CDSS). Such a tool can alert a clinician about an important event during a patient's treatment and allow for further investigation. In this case, it is important to also inform the clinician on the reasons behind the system's suggestion in order to make an informed decision.

Previous work in the investigation of QoL in ALS has investigated some predictors of QoL using linear models and simple statistics, while there are no machine learning-based predictive models or CDSS to support the faster prediction of QoL. Some scientists have found a correlation between physical functioning and QoL[35], while some contradict that [9, 18, 25, 28], or have only found relevance with the Physical Functioning QoL sub-scale[17] Regarding symptoms, dysphagia is an issue that relates to quality of life[24, 30], as well as breathing difficulties and fatigue[35]. Palliative care provided by the multidisciplinary team has been shown to improve QoL in two studies[4, 16]. In this work we have a rich set of information available and the support of machine learning techniques to assist us in gaining insight. In our previous work with the same cohort we predicted caregiver burden in carers of people with ALS, which is also a multi-dimensional outcome, and the results were promising[3].

The focus of this work is the development of a CDSS which will provide information on a patient's global QoL (low or high) along with explanations on the predicted outcome. The features used in the analysis contained patient and primary caregiver demographic information, along with use of health services, financial support, social status, aids and appliances received, expenses for treatment, clinical attributes of the patient and caregiving duties of the caregiver. The primary caregiver is the adult who took main responsibility for the patient by offering unpaid support. We believe that the caregiver plays an important role in the care of a patient with ALS, so some information about them might be related to the patient's QoL. Our goal is to create a system that will require a small number of easy to collect or readily-available inputs that will alert a clinician on the risk of a patient experiencing low levels of QoL, as awareness of this information is clinically useful[27]. We did not include other questionnaires in the study, for example, one that measures depression or anxiety which has been shown to affect QoL. Although this information is relevant their inclusion would have decreased the ease of information collection and clinical usability. An additional aim of our CDSS is to provide explanations for the reasons behind the alerts so that the clinicians can make an informed decision. Our proposed system will provide both global as well as local/individual explanations. This way clinicians will be aware and devise a personalised plan for the provision of the necessary support to the patient and their caregiver.

We are working towards the prediction of the overall quality of life that a patient experienced within the past two days as it is measured by the McGill QoL questionnaire[11]. There are two scores that quantify this, one is a single-item score (SIS), the self perceived QoL in the past two days, while the other (MQoL) is the mean value of the scores on five sub-scales that measure physical well-being, physical symptoms, existential well being, psychological symptoms and support. These two measures of QoL both describe global QoL and have been found to be highly correlated[28], but they are calculated in a different manner so it is useful for clinicians to investigate both.

\section{DATA}

This study included information on 90 people with ALS and their primary caregiver, who were interviewed at their residence at three time-points (at four-month to six-month intervals). The patients attended the National ALS/MND multidisciplinary Clinic (MDC) at Beaumont Hospital, Dublin. The pairs of participants were identified through the MDC and were asked to consider participating in the study. They provided informed consent for participation and for access to the patients' clinical information through the National ALS/MND Register after the follow-up interviews. All information was pseudonymised after collection and before conducting any analysis for this work. This study was granted ethical approval from Beaumont Hospital Medical Research Ethics Committee and all participants provided informed written consent.

The McGill Quality of Life (McGill) questionnaire was used to measure the quality of life on a scale of 0 ("very bad") to 10 ("excellent") for each question[11]. The stage of the disease for the patients was measured using both the Milano-Torino (MiToS) functional staging[10] and King's clinical staging[26] systems. The El Escorial[6] criteria were used to determine diagnosis. The Amyotrophic Lateral Sclerosis Functional Rating Scale-Revised (ALSFRSR)[7] was used as a measure of the progression of ALS. A high ALSFRS-R score shows a better functionality of the patient. The total score ranges between 0 and 48 and the individual questions (ranging between 0 and 4) quantify symptoms of the regions that are used in the El Escorial criteria. Cognitive and behavioural impairment were assessed by the Edinburgh Cognitive and Behavioural ALS Screen (ECAS)[1, 23] and Beaumont Behavioural Inventory (BBI)[12].

To calculate the total QoL in McGill, namely "MQoL", we used the method described in [11], while the "MQoL-SIS" score is a singleitem global measure of quality of life. The scores were then split in two classes according to their median value: 6 for MQoL-SIS and 6.85 for MQoL. A higher than median value is equivalent to high quality of life and is represented by 0 in our data, while a lower than median value is equivalent to a low quality of life and is represented by 1 (shown in Eq. 1). For the prediction of the two outcomes we used two datasets with the same features ( $\mathrm{N}=137$ predictors), but a different number of entries due to missingness. Entries with a missing value in the target feature were removed leaving 167 entries in the MQoL dataset and 176 entries in the MQoL-SIS dataset.

$$
\text { Binary QoL }= \begin{cases}1 \text { or low } & \text { QoL score } \leq \text { median value, } \\ 0 \text { or high } & \text { QoL score > median value. }\end{cases}
$$

\section{METHODS}

A summary of the methodology can be seen in the workflow diagram in Figure 1. This process was repeated for the two different outcomes in parallel. We then demonstrate the global and local explanations for the best models that predict MQoL, along with two case studies.

\subsection{Data Pre-processing}

In order to select a subset of the most relevant features we applied an ensemble of six different feature selection methods for the elimination of biases in the process[2]. This step was conducted in 


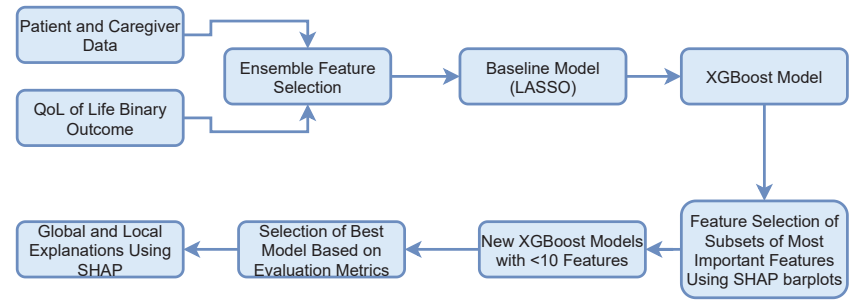

Figure 1: Workflow diagram that shows a summary of the methodology that was used in this paper. These steps were repeated for each of the two outcomes, MQoL and MQoL-SIS.

Table 1: Distribution of patients into classes. MQoL-SIS is the overall quality of life single-item Score while MQoL is the calculated total score based on the McGill QoL questionnaire.

\begin{tabular}{ccc}
\hline Data & High QoL(0) & Low QoL(1) \\
\hline$M Q o L-S I S$ & & \\
Full & 105 & 71 \\
Training & 81 & 51 \\
Test & 24 & 20 \\
\hline MQoL & & \\
Full & 85 & 82 \\
Training & 70 & 55 \\
Test & 15 & 27 \\
\hline
\end{tabular}

R (http://www.r-project.org/) using the "EFS" (Ensemble Feature Selection) package [22]. The ensemble consisted of the following methods: median, Pearson- and Spearman-correlation, logistic regression, and two variable importance measures embedded in the "randomForest" [5] implementation in R. From this methodology we identified the 50 and 51 important features for each of the two outcomes (MQoL and MQoL-SIS respectively).

\subsection{Data Analysis}

For the creation of the predictive models, the data were split into training and test sets (75\% and $25 \%$ respectively). The distribution of patients into QoL classes is shown in Table 1. Due to the lack of existing models for this research problem to compare ours against, we developed a baseline model using logistic regression; a technique that is explainable by design. A baseline model was trained for each of the two outcomes using Least Absolute Shrinkage and Selection Operator(LASSO)[32]. LASSO was used instead of a standard logistic regression because of the large number of features in the data. The lambda parameter that was used was identified using five-fold cross validation with the method cv.glmnet from the "glmnet" [14] package in $\mathrm{R}$ version 3.0-2. To create the model we used the function "glmnet" from the same package. For the baseline models (LASSO) we imputed the missing data using the missForest[29] imputation method in R, while the remaining models did not require the imputation of missing values.
Table 2: Hyperparameter values used in the grid search for the tuning of the XGBoost models.

\begin{tabular}{cc}
\hline Hyperarameter & Set of values \\
\hline eta & {$[0.001,0.01,0.1,0.2,0.3]$} \\
gamma & {$[0.05,0.5,1,1.5]$} \\
min_child_weight & {$[5,7,9,10]$} \\
subsample & {$[0.5,0.8,1]$} \\
colsample_bytree & {$[0.6,0.8,1]$} \\
lambda & {$[0.1,0.5,1]$} \\
\hline
\end{tabular}

We created the remaining models using the Extreme Gradient Boosting algorithm (XGBoost)[8]. XGBoost is a popular implementation of the gradient tree boosting method[13] that is easy to use, accurate and used by the winning teams of many Machine Learning competitions in different kinds of problems[8]. It creates an ensemble of Decision Trees gradually, by evaluating their predictions and introducing higher weights to the samples that were not successfully predicted in the previous tree, in order to prioritise them in the next prediction "attempt".

After creating the first models with the features identified by the EFS method, we selected their most important features as they were "revealed" by the Explainable Artificial Intelligence (XAI) methodology we describe in Section 3.3. This was done in order to create lower-dimensional datasets. Our aim is to use the least amount of information that can be informative for the prediction of the outcome. Fewer predictive features are easier to collect, so this aspect can lead to a more usable system. Moreover, according to the European General Data Protection Regulation's (GDPR) data minimisation principle, we should collect no more personal information than "is necessary in relation to the purposes for which they are processed"(https://gdpr-info.eu/art-5-gdpr/).

To tune each model's hyperparameters we used an exhaustive search over different values on a five-fold cross-validation, with the "GridSearchCV" from sklearn using the F1 score (scoring="f1") to evaluate the different parameter combinations. The hyperparameters that were tuned were the following: the step shrinkage to prevent overfitting ("eta"), the minimum loss reduction required to make a new split in a tree ("gamma"), the minimum sum of weights required in a child ("min_child_weight"), the subsample of samples to be selected for the creation of each tree ("subsample"), the subsample ratio of columns to create each tree ("colsample_bytree"), and the L2 Regularization term on weights ("lambda"). We set default values equal to 1000 for the number of estimators and 4 for the maximum depth of each Decision Tree in the ensemble. The set of values that were used in the grid search are shown in Table 2 and the final values of all hyperparameters in all models are presented in Table 3.

To evaluate the models we used the F1 score and recall on the training and test sets to see how they compare, and the precision and AUC-ROC (Area Under the Receiver Operator Characteristic Curve) on the test set. Recall is significant in this work as it is more important to accurately predict the people who need support - in this case, the low QoL class. 
Table 3: Hyperparameter tuning of predictive models. MQoL-SIS refers to the overall QoL Single-Item Score, while the MQoL refers to the the calculated total QoL. Where there is no number in the dataset name, the full dataset is implied, as it was selected by the feature selection method, while the numbers refer to the datasets with the 10, 5, 3 and 2 most important features. The number of estimators were 1000 and the maximum depth of each tree was set to 4 .

\begin{tabular}{|c|c|}
\hline Model & Hyperpameters \\
\hline MQoL-SIS Baseline & alpha: 1 , lambda: 0.0576 \\
\hline MQoL-SIS Full & colsample_bytree: 0.6 , eta: 0.1 , gamma: 0.05 , lambda: 0.1 , min_child_weight: 7 , subsample: 1 \\
\hline MQoL-SIS10 & colsample_bytree: 0.6 , eta: 0.3 , gamma: 1 , lambda: 0.5 , min_child_weight: 5 , subsample: 0.8 \\
\hline MQoL-SIS5 & colsample_bytree: 1 , eta: 0.2 , gamma: 0.5 , lambda: 0.1 , min_child_weight: 5 , subsample: 0.8 \\
\hline MQoL Baseline & alpha: 1 , lambda: 0.1186 \\
\hline MQoL Full & colsample_bytree: 0.8 , eta: 0.2 , gamma: 1 , lambda: 0.5 , min_child_weight: 5 , subsample: 0.8 \\
\hline MQoL10 & colsample_bytree: 0.8 , eta: 0.1 , gamma: 0.05 , lambda: 0.1 , min_child_weight: 5 , subsample: 1 \\
\hline MQoL5 & colsample_bytree: 0.8 , eta: 0.3 , gamma: 0.5 , lambda: 0.1 , min_child_weight: 5 , subsample: 1 \\
\hline MQoL3 & colsample_bytree: 0.8 , eta: 0.01 , gamma: 0.05 , lambda: 0.1 , min_child_weight: 5 , subsample: 1 \\
\hline MQoL2 & colsample_bytree: 0.6 , eta: 0.3 , gamma: 0.05 , lambda: 0.5 , min_child_weight: 5 , subsample: 0.8 \\
\hline
\end{tabular}

\subsection{Explainable AI}

As discussed in the Introduction, our aim is to create a system that can assist clinicians not only by predicting QoL, but by also explaining its predictions. Our baseline model uses a technique that is explainable by design and the remaining models we developed require post-hoc explanations. For this reason we used a state-ofthe-art explainable Artificial Intelligence (XAI) technique, namely SHAP (SHapley Additive exPlanations) [20]. SHAP values are suggested as a unified method of six other XAI methods to measure feature importance. This methodology offers the opportunity of both local explanations, for a specific prediction, as well as global explanations that summarise many local ones to give an overview of the model's structure. We used the Tree Explainer [19] as it provides optimal local explanations and interactions, as well as global insights in the structure of tree-based models, such as the XGBoost that we applied in this study. With regard to global explanations, we used local model summarization to present an overview of the model and feature importance.

\section{RESULTS}

In the first part of this section we present the evaluation of all the fitted models using F1 score, Precision, Recall and AUC ROC score. We show how well the two outcomes are predicted by the Baseline model that used LASSO Regression, the full model using XGBoost and the models that used subsets of features using XGBoost (Table 4).

The Baseline model for MQoL-SIS overfits the training data and the results in the training set are not good. The Full model is also overfitted on the training dataset with no good results in the test set. When a subset of ten or five most important features was used, we saw some improvement that would not suffice for the development of a CDSS.

Regarding the MQoL models, in the Baseline model that used LASSO Regression, the algorithm did not converge, the predictive model consists only of a constant value and predicts all outcomes as 0.476 , which means they were all classified as 0 . As a result, recall is 0 and the precision and F1 scores are NaN. However, when we
(A)

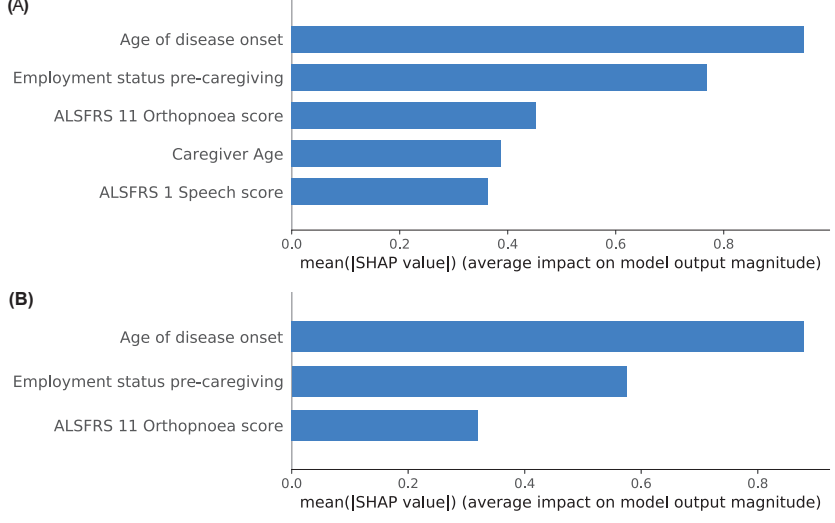

Figure 2: Bar plots that show features in order of importance based on the mean absolute value of the SHAP values for each feature in (A) MQoL5 and (B) MQoL3.

used the XGBoost algorithm the results were good on both the training and test sets, although in this case, the model underfits the training data. The good performance of the model that used only five features led us to remove more features and we found that three features were also quite predictive of the outcome. We are mostly interested in the prediction of the low QoL class $(\mathrm{MQoL}=1)$ in order to provide assistance to these patients, so higher recall is a priority. As a result we would recommend MQoL5 or MQoL3 (the models with only five or three features) as they use a very small number of features, while the recall in both the training and test sets is high. The recall in MQoL3 is slightly higher while the Precision is slightly lower compared to MQoL5.

\subsection{Model Explanations}

Due to their good predictive power, we are focusing on using the XAI techniques on models MQoL5 and MQoL3. Figure 2 shows a bar plot of the features in order of impact based on the mean absolute value of the SHAP values for each feature, to provide a 
Table 4: Evaluation of predictive models. MQoL-SIS refers to the overall QoL Single-Item Score, while the MQoL refers to the the calculated total QoL. The Baseline and "Full" models use the whole dataset, while the numbers next to the remaining model names indicate the number of features they use that were selected based on importance. AUC is the Area Under the ROC (Receiver Operator Characteristic) Curve.

\begin{tabular}{ccccccc}
\hline Model & f1-training & Recall-training & f1-test & Recall-test & Precision-test & AUC-test \\
\hline MQoL-SIS Baseline & 0.61 & 0.54 & 0.17 & 0.12 & 0.29 & 0.49 \\
MQoL-SIS Full & 0.54 & 0.90 & 0.41 & 0.35 & 0.50 & 0.53 \\
MQoL-SIS10 & 0.64 & 0.86 & 0.50 & 0.45 & 0.56 & 0.58 \\
MQoL-SIS5 & 0.61 & 0.82 & 0.53 & 0.45 & 0.64 & 0.62 \\
\hline MQoL Baseline & NaN & 0 & NaN & 0 & NaN & 0.50 \\
MQoL Full & 0.54 & 0.91 & 0.79 & 0.70 & 0.90 & 0.79 \\
MQoL10 & 0.66 & 0.96 & 0.80 & 0.74 & 0.87 & 0.77 \\
MQoL5 & 0.67 & 0.82 & 0.79 & 0.70 & 0.90 & 0.79 \\
MQoL3 & 0.62 & 0.84 & 0.81 & 0.78 & 0.84 & 0.76 \\
MQoL2 & 0.65 & 0.62 & 0.78 & 0.67 & 0.95 & 0.80 \\
\hline
\end{tabular}

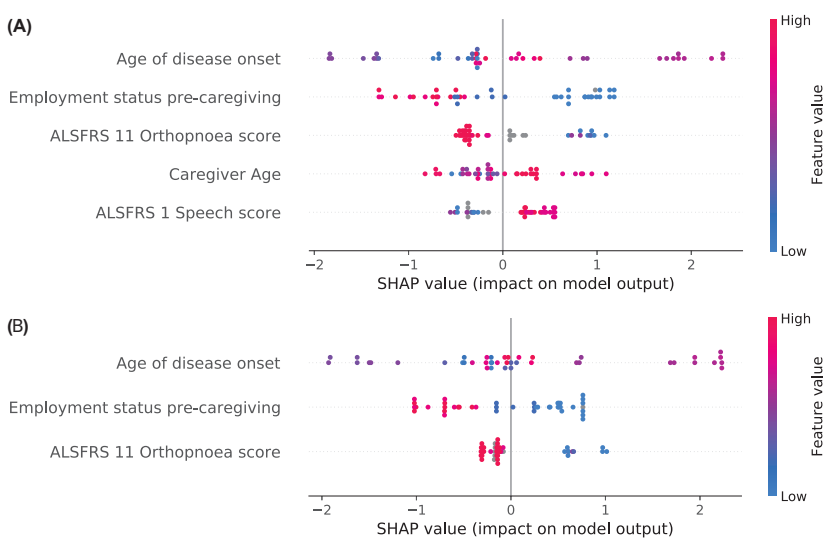

Figure 3: Summary plots of Models MQoL5(A) and MQoL3(B). Features appear in order of their sum of SHAP value magnitudes, and SHAP values show the impact each feature has on the model output. The color represents the feature value (red high, blue low). This reveals for example that a high ALSFRS-11 score for orthopnoea lowers the predicted risk of low QoL (or we could say that it is associated with higher QoL).

global explanation of the model. The patient's age at disease onset is the most important predictor with an average magnitude almost equal to 1 . It is followed by the caregiver's employment status prior to caregiving with almost 0.8 and then the remaining features have less magnitude although still important for the model. Figure 3 shows the summary plots of the two models. The features are displayed in order of importance, each of the dots is a different entry in the dataset and the color represents the feature value (red is high, blue is low). The reason some dots pile up vertically is to give a sense of density, and the reason they spread horizontally signifies their importance for some individual predictions, even if globally they might not be as important[19]. The plot is split by a vertical line on 0 , so all feature values on its left have a negative impact on the output while on its right they have a positive impact. If the colour on one side of the vertical line is mostly constant (close to red or close to blue) then it is easy to explain the relationship with the outcome. For instance, high values (good functionality) in the ALSFRS 11 orthopnoea score, which is a measure of shortness of breath when lying down, are predictive of higher QoL.

In order to explore the features some more we used dependence plots (Figure 4) to show how the model output varies by feature value (on the five-variable model for MQoL). The feature used for coloring is automatically chosen based on a potential interaction. In a similar manner to the summary plot, we can explain Figure 5 of local explanations for two hypothetical patients, one with a predicted QoL of 0 (the outcome in probabilities was equal to 0.33 ) and one with a predicted QoL of 1 (the outcome in probabilities was equal to 0.7 ). The features on the right (in blue) have a negative impact to the predicted outcome, while the red ones are "pushing" the predicted value to the right, meaning they have a positive impact. In this graph we can see the predicted probability, as well as the specific values the important features took in each individual case. It is important to note here that a "positive" impact is numerically positive, which means it moves the output value closer to 1 , which is equivalent to low QoL. In Section 5 we present the two hypothetical patients as case studies in order to put the CDSS in context.

\section{CASE STUDIES}

This section introduces two hypothetical patients along with their predicted probability of low QoL and the explanations of the prediction, in order to put the CDSS in context and to illustrate the factors that were predictive of their QoL. In Figure 5 we show the output of the 3-variable model for better visualisation using SHAP. This model uses the patient's age at disease onset and ALSFRS score for orthopnoea, as well as the caregiver's employment status before caregiving to make a prediction. A high orthopnoea score (4) corresponds to no breathing problems when lying flat, and a low score (0) represents inability to sleep without mechanical assistance. The caregiver's employment status is a categorical variable where: 1 represents "working for payment or profit"; 2 "looking for first regular job"; 3 "unemployed"; 4 "student/pupil”; 5 "looking after 


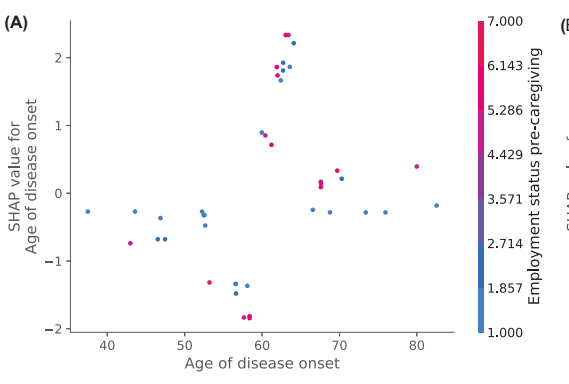

(D)

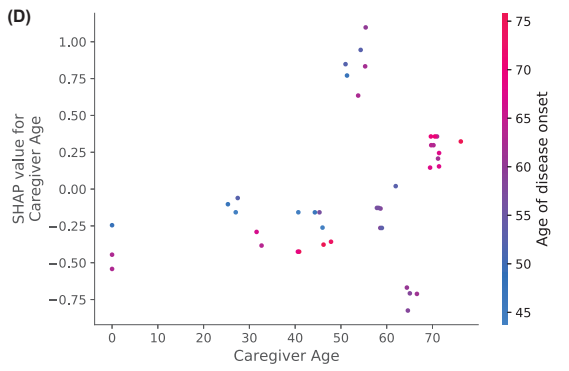

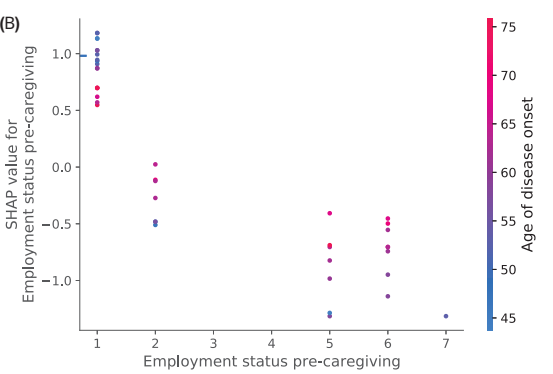
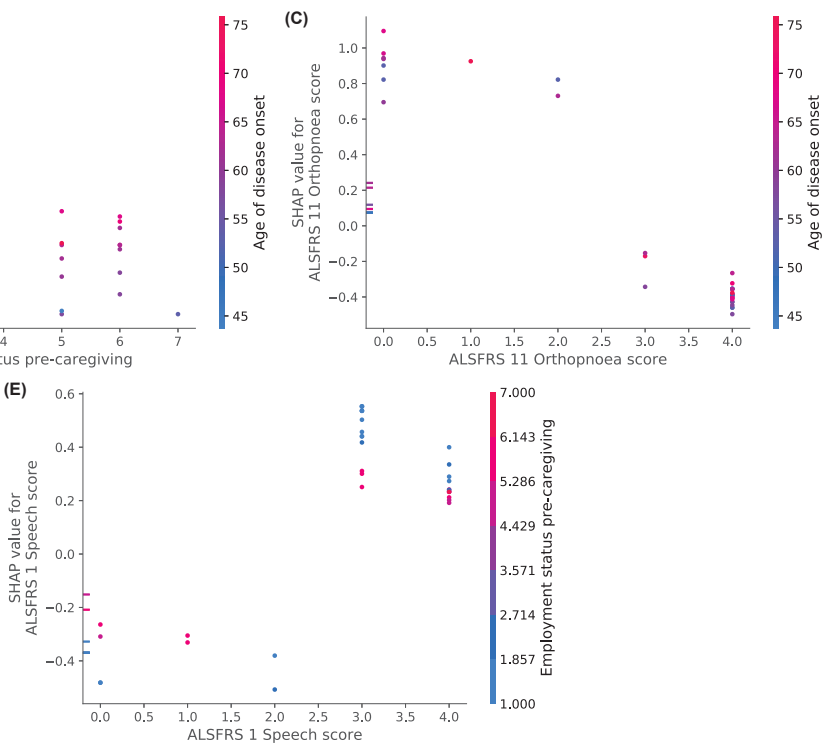

Figure 4: Dependence plots of features in MQoL5 (five-feature model for the prediction of MQoL) that show their impact on the outcome. Each dot is a different entry and the feature used for coloring is automatically chosen based on a potential interaction. (A) Age of onset, (B) Employment status pre caregiving, (C) ALSFRS 11 Orthopnoea score, (D) Caregiver Age, (E) ALSFRS 1 Speech, are all plotted against their SHAP value.

home/family"; 6 "retired from employment"; 7 "unable to work due to permanent sickness or disability"; and 8 "other".

Case 1. A 61-year-old Caucasian man, with no family history of ALS, presented at the multidisciplinary clinic 18 months after symptom onset, for his scheduled visit. He used to be a bank manager, but is now retired (not due to his ALS diagnosis). He was accompanied by his 63-year-old wife who, due to the fact that they had four children, used to look after the home/family; this is something she also does now, along with her caregiving duties. The patient's onset was spinal, and affected his movement, starting with weakness of his hips and legs. At this visit he was assessed and was found to need some assistance to perform his dressing and hygiene routine, and he also walks with assistance. He has no breathing, speaking or eating difficulties.

During the visit the patient's information is updated in the health records and based on the patient's age at disease onset, ALSFRS score for orthopnoea, and the caregiver's employment status before caregiving, the predicted probability for having a low QoL (a MQoL score below 6.85) is calculated. The patient has a predicted probability of low QoL equal to 0.33 , which means we would classify him as a low risk patient (or high QoL) at this time. The prediction and its explanation are shown in Figure 5(A). His age of onset was 60.4 and leads to an increase in the output value (as shown by the arrow and the red colour). The caregiver's employment status before caregiving was 5 (looking after home/family) and the ALSFRS 11 score for orthopnoea was 4 (high functionality). Both these features have a negative impact on the predicted probability. The caregiver's prior employment status is what, in this case, affected the prediction the most (based on the length of the blue arrow).
Case 2. A 66-year-old Caucasian woman, with no family history of ALS, presented at the multidisciplinary clinic five years after symptom onset, for her scheduled appointment. She continues having difficulty breathing and is using a BiPAP (Bilevel Positive Airway Pressure) during the night as she has respiratory insufficiency and orthopnoea. When she first attended the clinic, five years ago, she was a retired school teacher. She was accompanied to the clinic by her 68-year-old sister, who has also been retired for ten years. Her sister now lives with her because the patient needs assistance with walking, dressing and hygiene, and turning into bed, and she is her primary caregiver.

The predicted probability of low QoL for the patient was 0.70 which classifies her as an at-risk individual or a low QoL patient (Figure 5(B)). The age of onset was close to case 1 (61.2 years) and had a similar positive effect (although smaller), while the orthopnoea score was 0 (inability to sleep without mechanical assistance) and also has a positive impact on the predicted probability. The caregiver's prior employment status had a negative impact with a value of 6 (retired from employment). The feature that mostly affected the prediction in this case was the orthopnoea score.

\section{DISCUSSION}

The focus of this work was to develop a CDSS to alert clinicians to a patient's risk of low QoL. We used two different scores from the McGill questionnaire that represent overall QoL. MQoL-SIS, is defined as a single item score that describes overall self-perceived QoL in the past two days, while the MQoL score is calculated by sub-scores from the questionnaire that measure physical symptoms and well being, existential, psychological QoL, and support. We 

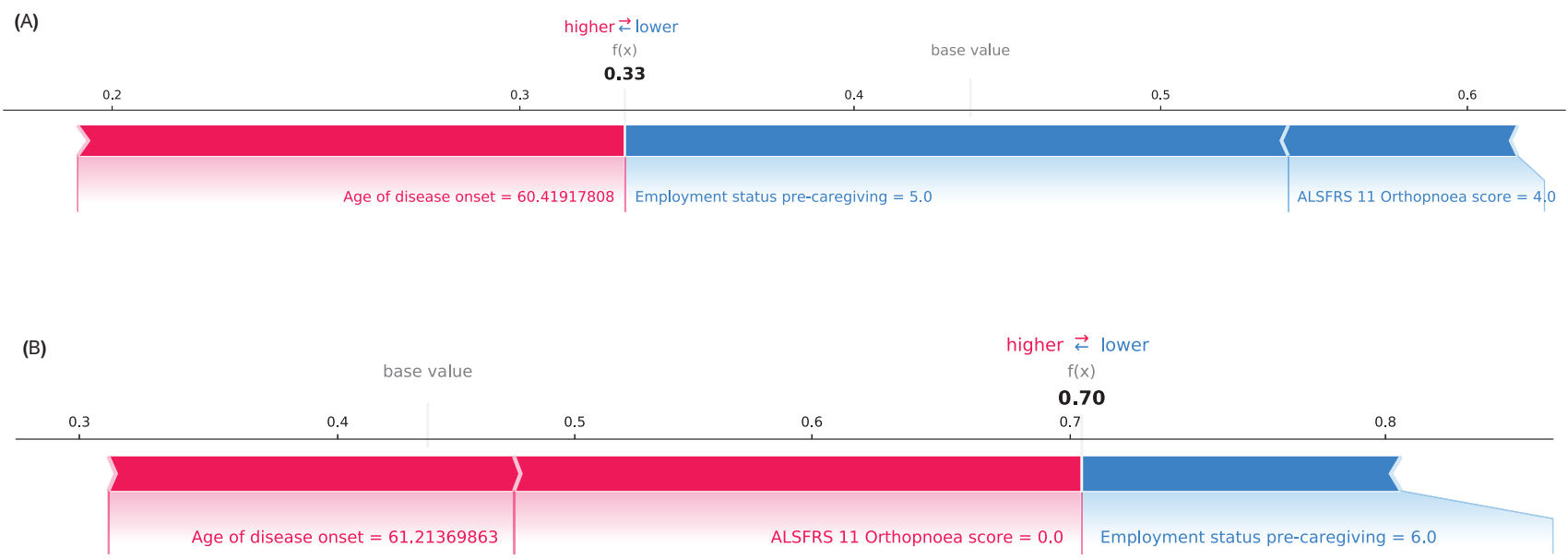

Figure 5: (A) Explanations on a patient's prediction of high MQoL. (B) Explanations on a patient's prediction of low MQoL. Both are created using model MQoL3. Blue shows a decrease in predicted outcome caused by the "blue" features, while the red show an increase. The outcome is shown in the form of predicted probability. A MQoL value of 1 means that we have a case (an "issue"), while 0 means non-case (or no "issue"). When a feature has a positive impact on the outcome it means that the predicted probability of the binary QoL score is increased and is "pushed" towards a value of 1; this value represents a low QoL score.

included information on the patient's clinical status, aids and appliances, use of healthcare services, expenses due to the condition, and demographic and financial status. Additionally, considering the patient and primary caregiver as a "system" that interacts, we included some demographic information on the caregiver, as well as caregiving duties, employment status and financial supports as a result of their relative's condition. We used an ensemble Machine Learning method, namely XGBoost, for the development of the models and an explainable technique, namely SHAP, to get insights for the predictions. We found that the SIS score is not easy to predict with the available information, while the calculated total MQoL score was predicted well by only a small number of variables. We identified two models with very similar scores and slight differences in the proportions of correct positive $(\mathrm{MQOL}=1)$ identifications. Evaluation on a larger number of patients could clarify whether there are significant differences in performance. The two selected models were the ones with three and five predictive features (MQoL3 and MQoL5) and they were selected over the two- and ten-feature models (MQoL2 and MQoL10) due to their good recall scores and small number of variables.

Our findings suggest that QoL (as it is measured by the Total MQoL score) is related to the patient's age at disease onset, orthopnoea score in the ALSFRS-R scale, and Speech Score in the ALSFRS-R scale. It is also related to the caregiver's age and employment status pre-caregiving. Using the dependence and summary plots we can further discuss the way each feature affects the predictions. It is important to note that the methodology does not reveal causality; rather, it is giving us insights into what affected the predicted value. Moreover, we emphasise the fact that a value of 1 means that we have a case (an "issue"), while 0 means non-case (or no "issue"). Thus, in this case, when a feature has a positive impact on the outcome it means that the predicted probability of the binary QoL score is increased and is "pushed" towards a value of 1 ; this value represents a low QoL score. So, a positive impact would be associated with lower QoL and vice versa.

With regards to MQoL, age of onset is the most predictive feature, but it affects the outcome in different ways. The highest ages (above 65 years) have a smaller absolute impact on QoL, while the smallest (below 55 years) have a small negative impact. Interestingly, the values in between have the biggest impact on QoL but it could be either positive or negative. This effect is not very clear and it could be associated with other factors, so we suggest further investigation. Regarding employment status pre-caregiving, we see that the primary caregiver being employed before the onset of their caregiving duties (value of 1 in the feature), has a positive impact on the outcome; thus, it is related to lower MQoL. This outcome might be associated to the caregiver's burden after the assignment to the additional duty of caregiving or to the satisfaction of the care the patient received. The latter has been found to be associated with MQoL in a previous study[9], along with social status. Lack of orthopnoea (high score) is associated with higher QoL and previous work has also found a similar effect on QoL due to breathing problems [35]. Higher speech functionality (ALSFRS 1) is related to lower QoL. In this case there could be a confounding factor that we are unaware of and this would require further investigation. It can be noted that the majority of patients in our study had small or no speech disturbances. Finally, we can see that a lower caregiver's age (below 50 years) is associated with higher QoL (has a negative 
impact on the predicted value). For ages above 50 there is variation of the effect.

We found that two of the differentiators of the QoL classes were caregiver-related information. Additional support for caregivers could potentially impact both their and the patient's QoL. Provision of palliative care has been found beneficial for the patient's QoL and for the alleviation of caregiver burden[4, 16]. Moreover, support to the family has been recommended for the improvement of QoL[15]. Social support and satisfaction of the care that patients receive affects QoL[9], so, awareness of a patient's QoL can lead to the timely provision of this support. Finally, younger patients might be less affected, according to our findings, but early support could maintain their levels of QoL.

This study is not without limitations, the main one being the small number of entries in our dataset due to the rarity of the disease. Evaluation of the models on more patients is recommended as a further step. Previous studies have found correlations between QoL and psychological, existential and spiritual factors[9, 25, 28, 33], which were not included in this work. Further investigation of such factors combined with the findings in this study could potentially explain some of the effects that were found, and even more accurately predict the MQoL-SIS Score. Moreover, social withdrawal and patient satisfaction for the care that patients receive were not measured in this study but have been identified as predictors of MQoL-SIS[9].

\section{CONCLUSION}

In this work we used a Machine Learning ensemble technique (XGBoost) to create a CDSS to alert clinicians about a patient's QoL in ALS. We also applied a state-of-the art technique which can explain the outputs to clinicians for better usability and system assessment. The explanations can be both individual and also related to the overall structure of the model. The most important predictors of QoL were the age at disease onset, ALSFRS score for orthopnoea and the caregiver's status pre-caregiving. Additionally caregiver age and ALSFRS score for speech can potentially add to the predictive power but this should be further evaluated on new patients. While the predictive features would require further investigation and a bigger sample would be needed to validate the predictive models, this work successfully predicts a patient's overall QoL, as it is defined by the calculated total QoL score (MQoL) from the McGill questionnaire. This study is a proof of concept of an informatics solution to assist in the easier assessment of a patient's QoL. We aim to validate these findings against a larger European ALS cohort and to evaluate the explainability of the CDSS with the help of clinicians.

\section{ACKNOWLEDGMENTS}

We would like to thank the patients and caregivers who participated in this research. This publication has emanated from research supported in part by a research grant from Science Foundation Ireland (SFI) under Grant Number 16/RC/3948 and co-funded under the European Regional Development Fund and by FutureNeuro industry partners. It was also made possible through funding from the Irish Health Research Board Dublin, (http://www.hrb.ie), as part of the HRB Interdisciplinary Capacity Enhancement Awards (ICE/2012/6) and HRB-JPND/2013/1 as well as by funding through the Clinical Management Grant from the American ALS Association (17 CM-324) (http://www.alsa.org).

\section{REFERENCES}

[1] Sharon Abrahams, Judith Newton, Elaine Niven, Jennifer Foley, and Thomas H. Bak. 2014. Screening for cognition and behaviour changes in ALS. Amyotrophic Lateral Sclerosis and Frontotemporal Degeneration 15, 1-2 (2014), 9-14. https://doi.org/10.3109/21678421.2013.805784 arXiv:https://doi.org/10.3109/21678421.2013.805784 PMID: 23781974.

[2] Anna Markella Antoniadi, Miriam Galvin, Mark Heverin, Orla Hardiman, and Catherine Mooney. 2020. Identifying features that are predictive of quality of life in people with amyotrophic lateral sclerosis. In 2020 IEEE International Conference on Healthcare Informatics (ICHI). 497-498.

[3] Anna Markella Antoniadi, Miriam Galvin, Mark Heverin, Orla Hardiman, and Catherine Mooney. 2020. Prediction of caregiver burden in amyotrophic lateral sclerosis: a machine learning approach using random forests applied to a cohort study. BMF Open 10, 2 (2020). https://doi.org/10.1136/bmjopen-2019-033109 arXiv:https://bmjopen.bmj.com/content/10/2/e033109.full.pdf

[4] Peter Bede, David Oliver, James Stodart, Leonard van den Berg, Zachary Simmons, Doiminic Ó Brannagáin, Gian Domenico Borasio, and Orla Hardiman. 2011. Palliative care in amyotrophic lateral sclerosis: a review of current international guidelines and initiatives. Fournal of Neurology, Neurosurgery \& Psychiatry 82, 4 (2011), 413-418. https://doi.org/10.1136/jnnp.2010.232637 arXiv:https://jnnp.bmj.com/content/82/4/413.full.pdf

[5] Leo Breiman. 2001. Random forests. Machine learning 45, 1 (2001), 5-32.

[6] Benjamin Rix Brooks. 1994. El escorial World Federation of Neurology criteria for the diagnosis of amyotrophic lateral sclerosis. Fournal of the Neurological Sciences 124 (1994), 96 - 107. https://doi.org/10.1016/0022-510X(94)90191-0

[7] Jesse M. Cedarbaum, Nancy Stambler, Errol Malta, Cynthia Fuller, Dana Hilt, Barbara Thurmond, and Arline Nakanishi. 1999. The ALSFRS-R: a revised ALS functional rating scale that incorporates assessments of respiratory function. fournal of the Neurological Sciences 169, 1 (1999), 13 - 21. https://doi.org/10.1016/ S0022-510X(99)00210-5

[8] Tianqi Chen and Carlos Guestrin. 2016. XGBoost: A Scalable Tree Boosting System. In Proceedings of the 22nd ACM SIGKDD International Conference on Knowledge Discovery and Data Mining (KDD '16). Association for Computing Machinery, New York, NY, USA, 785-794. https://doi.org/10.1145/2939672.2939785

[9] Adriano Chiò, A Gauthier, Anna Montuschi, Andrea Calvo, N Di Vito, Paolo Ghiglione, and Roberto Mutani. 2004. A cross sectional study on determinants of quality of life in ALS. Fournal of Neurology, Neurosurgery \& Psychiatry 75, 11 (2004), 1597-1601.

[10] Adriano Chiò, Edward R Hammond, Gabriele Mora, Virginio Bonito, and Graziella Filippini. 2015. Development and evaluation of a clinical staging system for amyotrophic lateral sclerosis. Fournal of Neurology, Neurosurgery \& Psychiatry 86, 1 (2015), 38-44. https://doi.org/10.1136/jnnp-2013-306589

[11] S. Robin Cohen. 2014. McGill Quality of Life Questionnaire. Springer Netherlands, Dordrecht, 3883-3889. https://doi.org/10.1007/978-94-007-0753-5_1749

[12] Marwa Elamin, Marta Pinto-Grau, Tom Burke, Peter Bede, James Rooney, Meabhdh O’Sullivan, Katie Lonergan, Emma Kirby, Emma Quinlan, Nadia Breen, Alice Vajda, Mark Heverin, Niall Pender, and Orla Hardiman. 2017. Identifying behavioural changes in ALS: Validation of the Beaumont Behavioural Inventory (BBI). Amyotrophic Lateral Sclerosis and Frontotemporal Degeneration 18, 1-2 (2017), 68-73. https://doi.org/10.1080/21678421.2016.1248976 arXiv:https://doi.org/10.1080/21678421.2016.1248976 PMID: 27894191

[13] Jerome Friedman. 2001. Greedy Function Approximation: A Gradient Boosting Machine. Annals of Statistics 29 (10 2001), 1189-1232. https://doi.org/10.2307/ 2699986

[14] Jerome Friedman, Trevor Hastie, and Rob Tibshirani. 2010. Regularization Paths for Generalized Linear Models via Coordinate Descent. Fournal of Statistical Software, Articles 33, 1 (2010), 1-22. https://doi.org/10.18637/jss.v033.i01

[15] Birgitta Jakobsson Larsson, AG Ozanne, Karin Nordin, and Ingela Nygren. 2017. A prospective study of quality of life in amyotrophic lateral sclerosis patients. Acta Neurologica Scandinavica 136, 6 (2017), 631-638.

[16] Chafic Y Karam, Sabrina Paganoni, Nanette Joyce, Gregory T Carter, and Richard Bedlack. 2016. Palliative care issues in amyotrophic lateral sclerosis: an evidencedbased review. American fournal of Hospice and Palliative Medicine® 33, 1 (2016), 84-92.

[17] Sonja Körner, Katja Kollewe, Susanne Abdulla, Antonia Zapf, Reinhard Dengler, and Susanne Petri. 2015. Interaction of physical function, quality of life and depression in Amyotrophic lateral sclerosis: characterization of a large patient cohort. BMC neurology 15, 1 (2015), 1-8.

[18] Dorothée Lulé, Sonja Häcker, Albert Ludolph, Niels Birbaumer, and Andrea Kübler. 2008. Depression and quality of life in patients with amyotrophic lateral sclerosis. Deutsches Ärzteblatt International 105, 23 (2008), 397.

[19] Scott M. Lundberg, Gabriel Erion, Hugh Chen, Alex DeGrave, Jordan M. Prutkin, Bala Nair, Ronit Katz, Jonathan Himmelfarb, Nisha Bansal, and Su-In Lee. 2020. 
From local explanations to global understanding with explainable AI for trees. Nature Machine Intelligence 2, 1 (2020), 2522-5839.

[20] Scott M Lundberg and Su-In Lee. 2017. A Unified Approach to Interpreting Model Predictions. In Advances in Neural Information Processing Systems 30, I. Guyon, U. V. Luxburg, S. Bengio, H. Wallach, R. Fergus, S. Vishwanathan, and R. Garnett (Eds.). Curran Associates, Inc., 4765-4774. http://papers.nips.cc/paper/ 7062-a-unified-approach-to-interpreting-model-predictions.pdf

[21] Janet E McLeod and David M Clarke. 2007. A review of psychosocial aspects of motor neurone disease. Journal of the neurological sciences 258, 1-2 (2007), 4-10.

[22] Ursula Neumann, Nikita Genze, and Dominik Heider. 2017. EFS: an ensemble feature selection tool implemented as R-package and web-application. BioData mining 10, 1 (2017), 1-9.

[23] Elaine Niven, Judith Newton, Jennifer Foley, Shuna Colville, Robert Swingler Siddharthan Chandran, Thomas H. Bak, and Sharon Abrahams. 2015. Validation of the Edinburgh Cognitive and Behavioural Amyotrophic Lateral Sclerosis Screen (ECAS): A cognitive tool for motor disorders. Amyotrophic Lateral Sclerosis and Frontotemporal Degeneration 16, 3-4 (2015), 172-179. https://doi.org/10.3109/ 21678421.2015.1030430 arXiv:https://doi.org/10.3109/21678421.2015.1030430

[24] G. Paris, O. Martinaud, A. Petit, A. Cuvelier, D. Hannequin, P. Roppeneck, and E. Verin. 2013. Oropharyngeal dysphagia in amyotrophic lateral sclerosis alters quality of life. Journal of Oral Rehabilitation 40, 3 (2013), 199-204. https://doi.org/10 1111/joor.12019 arXiv:https://onlinelibrary wiley.com/doi/pdf/10.1111/joor.12019

[25] R.A. Robbins, Z. Simmons, B.A. Bremer, S.M. Walsh, and S. Fischer 2001. Quality of life in ALS is maintained as physical function declines. Neurology 56, 4 (2001), 442-444. https://doi.org/10.1212/WNL.56.4.442 arXiv:https://n.neurology.org/content/56/4/442.full.pdf

[26] Jose C. Roche, Ricardo Rojas-Garcia, Kirsten M. Scott, William Scotton, Catherine E. Ellis, Rachel Burman, Lokesh Wijesekera, Martin R. Turner, P. Nigel Leigh, Christopher E. Shaw, and Ammar Al-Chalabi. 2012. A proposed staging system for amyotrophic lateral sclerosis. Brain 135, 3 (2012), 847-852. https://doi.org/10.1093/brain/awr351
[27] Zachary Simmons. 2015. Patient-perceived outcomes and quality of life in ALS. Neurotherapeutics 12, 2 (2015), 394-402.

[28] Zachary Simmons, Barbara A Bremer, Rosemary A Robbins, Susan M Walsh, and Stephan Fischer. 2000. Quality of life in ALS depends on factors other than strength and physical function. Neurology 55, 3 (2000), 388-392.

[29] Daniel J. Stekhoven and Peter Bühlmann. 2011. MissForest-non-parametric missing value imputation for mixed-type data. Bioinformatics 28, 1 (2011), 112118. https://doi.org/10.1093/bioinformatics/btr597

[30] Lauren Tabor, Joy Gaziano, Stephanie Watts, Raele Robison, and Emily K Plowman. 2016. Defining swallowing-related quality of life profiles in individuals with amyotrophic lateral sclerosis. Dysphagia 31, 3 (2016), 376-382.

[31] Evelyn O. Talbott, Angela M Malek, and David Lacomis. 2016. The epidemiology of amyotrophic lateral sclerosis. Handbook of clinical neurology 138 (2016), 225-38. https://doi.org/10.1016/B978-0-12-802973-2.00013-6

[32] Robert Tibshirani. 1996. Regression Shrinkage and Selection via the Lasso. Fournal of the Royal Statistical Society. Series B (Methodological) 58, 1 (1996), 267-288. http://www.jstor.org/stable/2346178

[33] A. Vignola, A. Guzzo, A. Calvo, C. Moglia, A. Pessia, E. Cavallo, S. Cammarosano, S. Giacone, P. Ghiglione, and A. Chiò. 2008. Anxiety undermines quality of life in ALS patients and caregivers. European fournal of Neurology 15, 11 (2008), 1231-1236. https://doi.org/10.1111/j.1468-1331.2008.02303.x arXiv:https://onlinelibrary.wiley.com/doi/pdf/10.1111/j.1468-1331.2008.02303.x

[34] Lokesh C Wijesekera and P Nigel Leigh. 2009. Amyotrophic lateral sclerosis. Orphanet journal of rare diseases 4, 1 (2009), 3.

[35] Carolyn Anne Young, John Ealing, Christopher McDermott, Tim Williams, Ammar Al-Chalabi, Tahir Majeed, Georgina Burke, Ashwin Pinto, David Dick, Kevin Talbot, et al. 2019. The relationships between symptoms, disability, perceived health and quality of life in amyotrophic lateral sclerosis/motor neuron disease. Amyotrophic Lateral Sclerosis and Frontotemporal Degeneration 20, 5-6 (2019), 317-327. 